\title{
Ship detection in strong clutter environment based on adaptive regression thresholding for HFSWR
}

\author{
Ji Yonggang ${ }^{1, \text { a }}$,Xu Leda ${ }^{1, b}$,Wang Yiming ${ }^{1, c}$ and Chu Xiaoliang ${ }^{1, d}$ \\ ${ }^{1}$ First Institute of Oceanography, State Oceanic Administration, No.6 Xianxialing Road, Qingdao, \\ CHINA \\ ajiyonggang@gmail.com, ${ }^{\mathrm{b} x u l e d a @ 126 . c o m,},{ }^{\mathrm{C}}$ wym.fio@gmail.com, ${ }^{\mathrm{d}} \mathrm{xlchu@ouc.edu.cn}$
}

Keywords:HFSWR; ship detection; strong clutter environment; adaptive detection method

\begin{abstract}
High Frequency Surface Wave Radar (HFSWR) has the ability to detect and track ships in real time and beyond the horizon continuously. The method of adaptive power regression thresholding (APRT) is one of good adaptive detection methods for HFSWR, and it works well in most cases. But in strong clutter environment, some strong clutters with high amplitude will raise the noise level, which would overestimate the detection threshold along Doppler cells or range cells and cause some ships become undetectable. This paper proposes an improved method to solve the problem appearing in strong clutter environment based on APRT method. It adds a process of strong clutter suppression before ship detection to eliminate its influence on estimation of noise level and detection threshold. The processing results of real measured HFSWR data show that the improved method can effectively improve the performance of detector under strong sea clutter environment.
\end{abstract}

\section{Introduction}

For ship detection with HFSWR, CA-CFAR and OS-CFAR are two commonly used detector of constant false alarm (CFAR). CA-CFAR is one of the simplest detectors, which can be easily realized. It uses the average of reference units to estimate the noise level anddetection threshold, so it is only suitable for ship detection in uniform background $[1,2]$. OS-CFAR calculates the detection threshold by use the value of a specific location of the sorted reference units.It can overcome the effect of some clutter and interference and have good robustness [3,4]. But its processing speed is slow because of the sorting process of reference units and detection preference become worse in strong clutter environment.Dzvonkovskaya and Rohlingproposed an adaptive detection algorithm for HFSWR, i.e. APRT method, which is based on the adaptive power regression thresholding separately for Doppler frequency and range cells[5,6]. The method of APRTcan solve the target detection problem in a complex environment and can work well in most cases. But in strong clutter environment, such as in high sea state or when many ships appear in the same range cell with different radial velocity or in the same Doppler frequency cell with different range. The noise level and corresponding detection threshold would be overestimated. Then, some ships would be undetected if its echo amplitude is lower than the overrated detection threshold, which can result in higher misdetection rate.So theappearance of strong clutters has a great influence on detection result of the APRT method, and new detection method for HFSWR should be studied in strong noise environment.

In this paper, an improved method was proposed to solve the problem of ship detection for HFSWR in strong clutter environmenton the basis of theAPRTmethod. This paper is structured as follows: Section 2 introduces the principle and processing flow of the new proposed method. Section 3 demonstrates the validation of the proposed method with experimental data. Section 4 concludes the paper.

\section{Principle and process flowchart}

In strong clutter environment, the main reason for the increase of misdetection rate is thathigh echo amplitude of strong clutters will raise the noise level and the detection threshold. In order to obtain better detection results, we should reduce the influence of strong clutters before the process of 
detection threshold estimation. The signal process flowchart of the improved detection method in strong clutter environment is shown in Fig. 1.

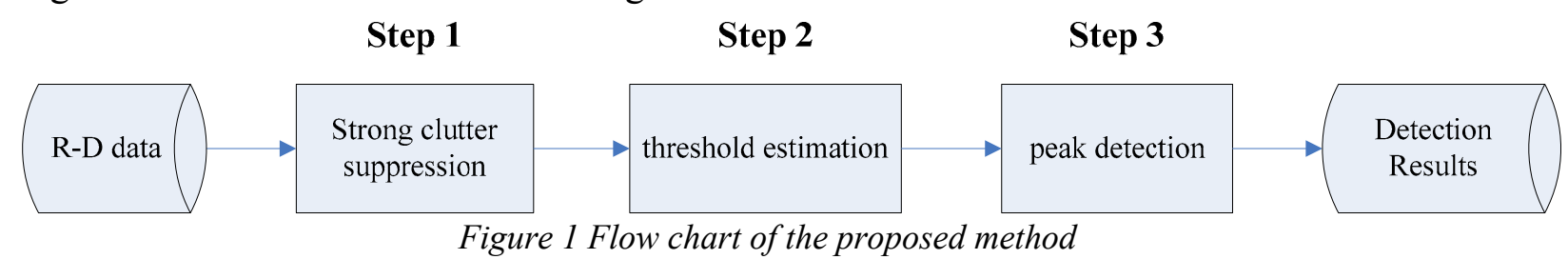

The improved detection method involves three steps. The first step is strong clutter suppression. The second step is threshold estimation. And the last step is peak detection.

Step1:Strong clutter suppression is the key step of the method. As the strong clutter can affect both the spectrum datain range dimension and data inDopplerdimension, clutter suppression should be performed on both space dimensions. For spectrum data of HFSWR $y(i)$, a curvilinear regression analysis can be used to get a preliminary estimate of noise level $f(i)$, and here $i$ is the index of rangeor Doppler cell. The threshold of strong clutter is $\operatorname{set} k f(i) . k$ is coefficient, which can be adjusted according to the amplitude and distribution of clutter. The echoes, whose amplitude are higher thanthe threshold of strong clutterk $f(i)$, will be treated as strong clutter and their amplitudes are replaced withnoise level $f(i)$ according to Eq. 1.Thus the strong clutter can be suppressed through the above pretreatment process.

$$
\left\{\begin{array}{l}
y(i)=f(i), \text { when } y(i) \geq k f(i) \\
y(i)=y(i), \text { when } y(i)<k f(i)
\end{array} .\right.
$$

Step2:After strong clutter suppression, the method of APRT can be applied on preprocessed data to obtain suitable detection threshold. For spectrum data along range cells, a curvilinear regression of 2nd order polynomial is used to estimate true value of noise level and detection threshold $T_{\text {range }}$. For spectrum data along Doppler cells, a curvilinear regression of 3nd order polynomial rather than 2nd order polynomial can be used to get detection threshold along Doppler cells $T_{\text {Doppler }}$. As neither $T_{\text {range }}$ nor $T_{\text {Doppler }}$ is suitable to all range-Doppler cells, an adaptive threshold $T$ should be estimated. Its value can choose the bigger one between $T_{\text {range }}$ and $T_{\text {Doppler }}$, which can be obtained by Eq. 2 .

$$
T=\max \left\{T_{\text {range }}, T_{\text {Doppler }}\right\} .
$$

After that, the estimated thresholdcan be applied on the original data rather than the preprocessed data to detect the vessels in Rang-Doppler image.If the amplitude of an echo is higher than the threshold $T$, it can be recognized as a target, or it is determined as background. See Eq. 3. In this step, the detection result is area targets rather than point targets.

$$
\left\{\begin{array}{l}
y(i)>T, \quad \text { target } \\
y(i) \leq T, \text { background }
\end{array}\right.
$$

Step3:Peak detection is to obtain the accurate position in the two-dimensional space of Rang-Doppler [7]. For HFSWR data, a $3 \times 3$ window can be used to obtain the peak points in range-Doppler image. Then after peak detection, the point targets can be obtained from the detection results of area targets.

\section{Detection analysis with measure data}

The HFSWR used in this paper is $4.7 \mathrm{MHz}, 8$ receive array antennas, and the coherent accumulation time is about 300s. Fig. 2 shows the estimation of noise level and clutter threshold by using the data long range cells at 133th Doppler bin. Fig. 3 show the preprocessed result after strong clutter suppression. As we can see that most strong clutters, whose amplitude are higher than the threshold, have been eliminated. In fact, those so-called strong clutters actually contain some ships. 


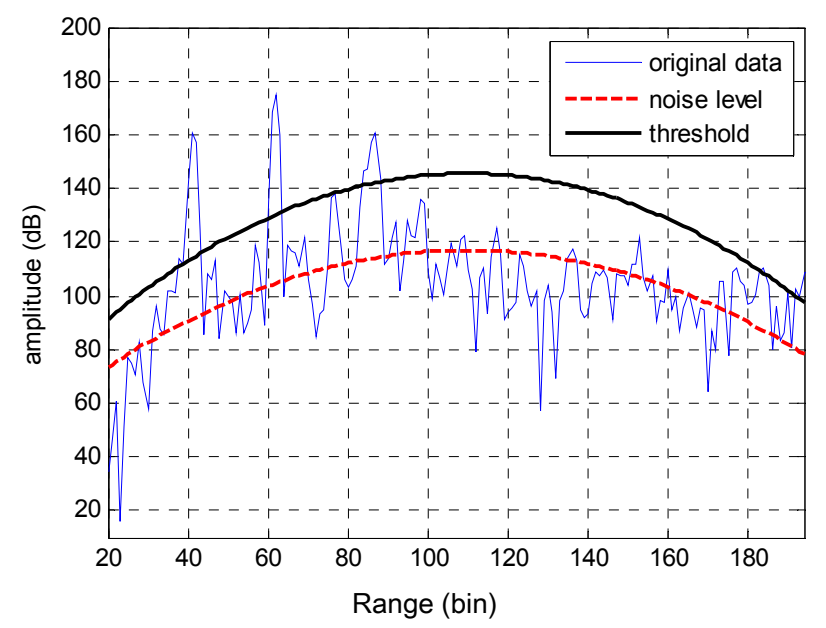

Figure 2 preliminary estimation of noise level and threshold along range cells at 133 Doppler

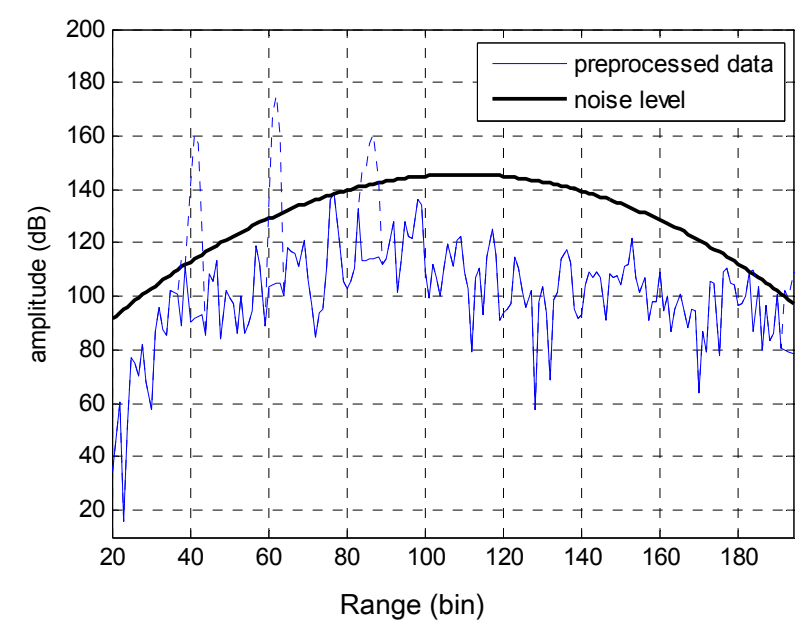

Figure 3 preprocessed data after strong clutter suppression

Fig. 4 and Fig. 5 show the detection results by using APRT method and improved APRT method respectively. The red crosses indicate the position of detectedvessels of point targets.As can be seen from Fig. 4a and Fig. 5a that there are more vessels detected by using improve APRT method than by using APRT method.

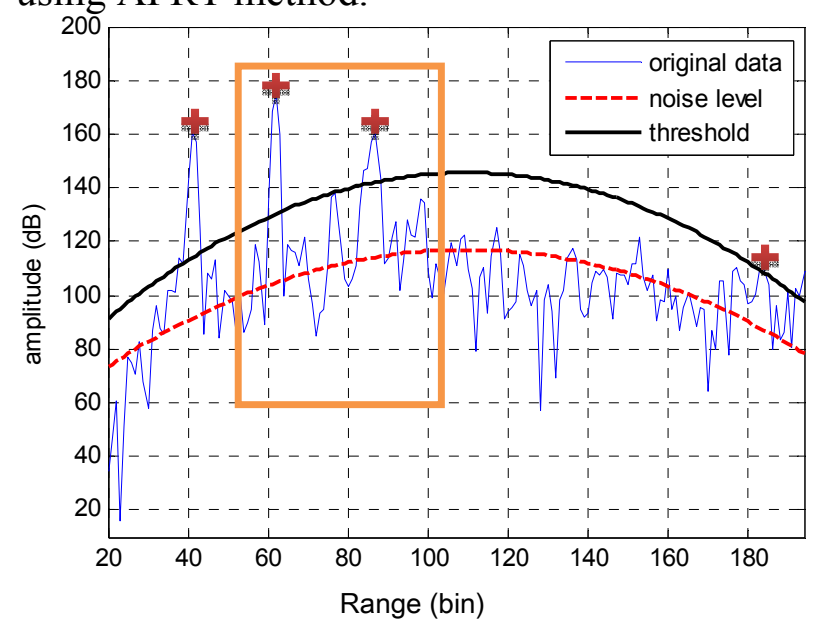

a. Detection result of APRT method

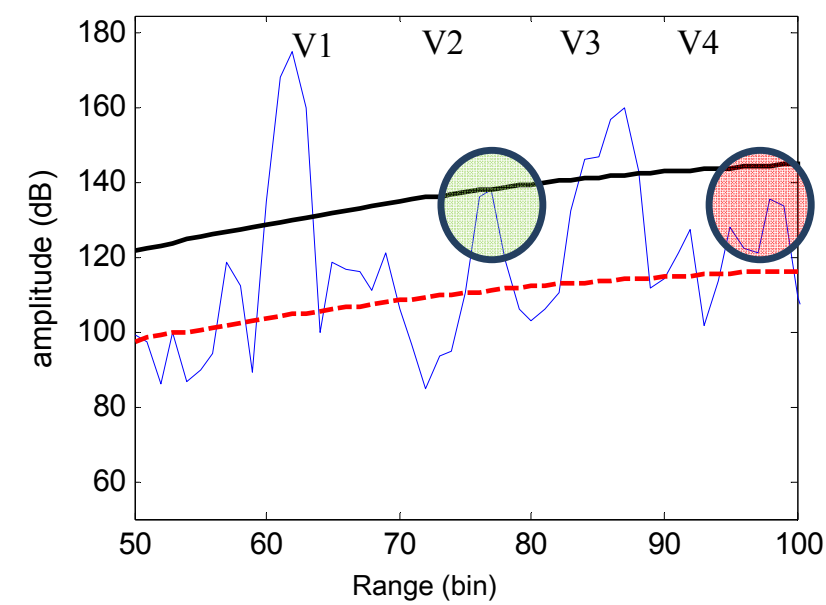

b. Enlarge Figure

Figure 4 detection result of APRT method along range cells at 133 Doppler

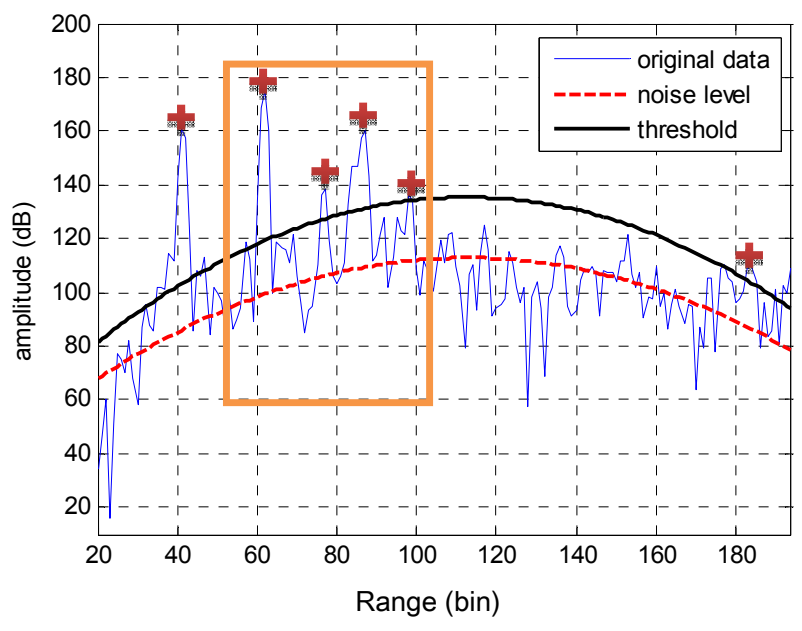

a. Detection result of improved APRT method

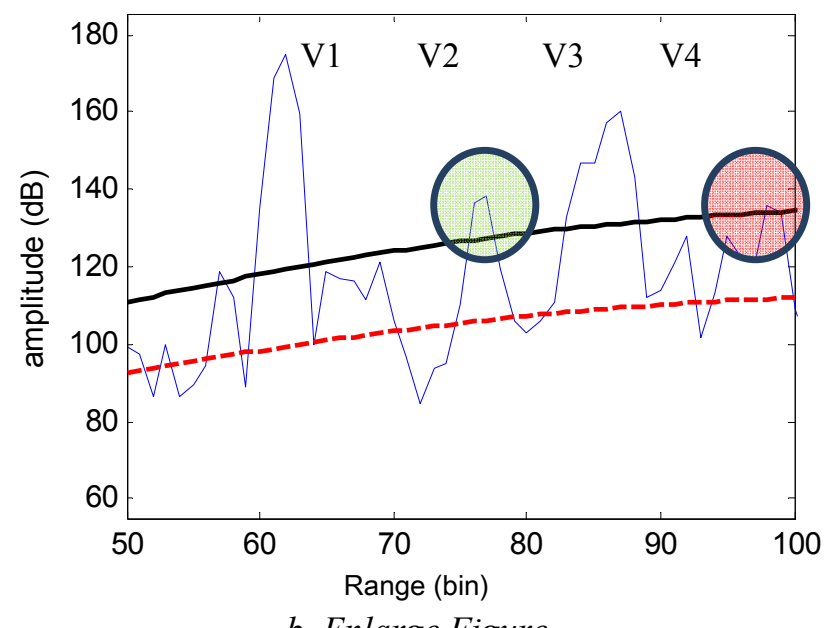

b. Enlarge Figure

Figure 5 detection result of improved APRT method along range cells at 133 Doppler

Fig. 5billustratesthat target V2 and V4 can be detected in the detection results of improved APRT method. But they are not detectedwhen the APRT method was used. As both the adjacent target V1 and V3 have high echo amplitudes, they can overestimate the noise level and detection threshold.As vessels V2 and V4 have weaker echo amplitudes, whose values are lower than the estimated threshold, 
they will be missed in such situation. When the effect of V1 and V3 has been eliminated when the improved APRT method was used, it would give good detection results.

\section{Conclusion}

In this paper, an improved APRTmethod was proposed to solve the problem of ship detection in strong clutter environment. The detection results of real measured HFSWR data show that the improved method can effectively improve the performance of detector under strong sea clutter environment.

In some cases, the improved method can also improve the detection performance under other environments, such as when multiple targets or ionosphere background appear. And those research or verification work will be carried out in the future.

\section{Acknowledgments}

This work was supported by the Marine scientific research special funds for public welfare (201505002) and the Special funds for Fundamental Research Project (0213T03).

\section{References}

[1] M.W.Long and R.A.Llamas: CA-CFAR performance with linear, square-law, and fourth-power detectors, Radar Conference (RADAR), 2011 IEEE. IEEE(2011), p. 350-355.

[2] W.Liu and J.S.Fu: A new method for analysis of M-pulses CA-CFAR in Weibull background, Radar-2000- The record of the IEEE International Radar Conference, Alexandria, VA(2001), p. 509-514.

[3] C.M. Cho and M.Barkat: Moving ordered statistics CFAR detection for nonhomogeneous backgrounds, IEE Proceedings F (Radar and Signal Processing). IET Digital Library, Vol. 140, No. 5 (1993), p.284-290.

[4] M. Shor and N.Levanon: Performances of order statistics CFAR, Aerospace and Electronic Systems, IEEE Transactions on, Vol. 27, No. 2 (1991), p. 214-224.

[5] A.L. Dzvonkovskaya andR.Hermann: Ship Detection with Adaptive Power Regression Thresholding for HF Radar, Radar Science and Technology, Vol. 2 (2007), p.81-85.

[6] A.L.Dzvonkovskaya and H.Rohling: Target detection with adaptive power regression thresholding for HF radar, Radar, 2006. CIE'06. International Conference on. IEEE(2006), p.1-4.

[7] S.J. Davey, S. B. Colegrove and D.Mudge: Advanced Jindalee Tracker: Enhanced Peak Detector, Defence Science and Technology Organization (Australia), Vol. 6 (1999), p. 2 7. 